\title{
Voice Handicap Index and Voice-Related Quality of Life in Idiopathic Parkinson's Disease
}

\section{Gyung Yu, Insoo Jang, Lakhyung Kim}

J of Oriental Neuropsychiatry 2013:24(2):155-162, http://dx. doi.org/10.7231/jon.2013.24.2.155

Added the abbreviation explanation of the " $\mathrm{M} \pm \mathrm{S}$. D"

Table 1. General Characteristics and UPDRS, VHI-10, VRQOL Scores

\begin{tabular}{lc}
\hline & $\mathrm{M} \pm \mathrm{S} . \mathrm{D}$ \\
\hline Age & $62.65 \pm 7.54$ \\
Med-Duration & $7.60 \pm 5.19$ \\
\hline UPDRS I & $3.59 \pm 2.48$ \\
UPDRS II & $16.00 \pm 6.99$ \\
UPDRS III & $21.59 \pm 9.12$ \\
\hline VHI-10 & $14.35 \pm 8.07$ \\
VRQOL-S & $59.93 \pm 20.50$ \\
VRQOL-P & $58.58 \pm 21.77$ \\
VRQOL-T & $59.12 \pm 20.25$ \\
\hline
\end{tabular}

Med-duration: years after medication, UPDRS: Unified Parkinson's Disease Rating Scale, VHI-10: Voice Handicap Index-10, VRQOL-S: Voice-Related Quality of Life social-emotional, P: physical functioning, T: total score.
Table 1. General Characteristics and UPDRS, VHI-10, VRQOL Scores

\begin{tabular}{lc}
\hline & $\mathrm{M} \pm \mathrm{S} . \mathrm{D}$ \\
\hline Age & $62.65 \pm 7.54$ \\
Med-Duration & $7.60 \pm 5.19$ \\
\hline UPDRS I & $3.59 \pm 2.48$ \\
UPDRS II & $16.00 \pm 6.99$ \\
UPDRS III & $21.59 \pm 9.12$ \\
\hline VHI-10 & $14.35 \pm 8.07$ \\
VRQOL-S & $59.93 \pm 20.50$ \\
VRQOL-P & $58.58 \pm 21.77$ \\
VRQOL-T & $59.12 \pm 20.25$ \\
\hline
\end{tabular}

$\mathrm{M} \pm$ S.D: Mean \pm Standard Deviation, Med-duration: years after medication, UPDRS: Unified Parkinson's Disease Rating Scale, VHI-10: Voice Handicap Index-10, VRQOL-S: Voice-Related Quality of Life social-emotional, P: physical functioning, T: total score. 


\section{The Differences of Learning Characteristics in Sasang Constitution}

Woo-Chang Choi, Woo-Kyoung Kim, Jeong-Mo Song, Lak-Hyung Kim

J of Oriental Neuropsychiatry 2013:24(2):163-178, http://dx.doi.org/10.7231/jon.2013.24.2.163

The word "N" in the legend of Table 1 should have been written as "Number"

Table 1. General Characteristics of Participants

\begin{tabular}{lccccc}
\hline & Soyangin & Taeeumin & Soeumin & Total & p-value \\
\hline $\mathrm{N}$ & $43(34.40)$ & $45(36.00)$ & $37(29.60)$ & $125(100.0)$ & \\
Age (years) & $24.86 \pm 3.649^{*}$ & $24.76 \pm 4.307$ & $24.92 \pm 4.078$ & $24.84 \pm 3.991$ & $0.983^{\dagger}$ \\
Gender (M/F) & $16 / 27$ & $26 / 19$ & $16 / 21$ & $58 / 67$ & $0.137^{\ddagger}$ \\
\hline
\end{tabular}

Values are number (\%).

*Values are mean \pm standard deviation, ${ }^{\dagger}$ One way ANOVA, ${ }^{\dagger}$ Pearson chi-square test.

Table 1. General Characteristics of Participants

\begin{tabular}{lccccc}
\hline & Soyangin & Taeeumin & Soeumin & Total & p-value \\
\hline Number & $43(34.40)$ & $45(36.00)$ & $37(29.60)$ & $125(100.0)$ & \\
Age (years) & $24.86 \pm 3.649^{*}$ & $24.76 \pm 4.307$ & $24.92 \pm 4.078$ & $24.84 \pm 3.991$ & $0.983^{\dagger}$ \\
Gender $(\mathrm{M} / \mathrm{F})$ & $16 / 27$ & $26 / 19$ & $16 / 21$ & $58 / 67$ & $0.137^{\ddagger}$ \\
\hline
\end{tabular}

Values are number (\%).

*Values are mean \pm standard deviation, ${ }^{\dagger}$ One way ANOVA, ${ }^{\ddagger}$ Pearson chi-square test. 
Added the abbreviation explanation of the "ASEF", "AFTT"

Table 3. Subscales Results of Academic Motivation Tests for each Sasang Constitutional Type

\begin{tabular}{ccccccc}
\hline & & Soyangin & Taeeumin & Soeumin & Total & p-value* \\
\hline \multirow{2}{*}{ ASET } & SEC & $3.80 \pm 0.54$ & $3.64 \pm 0.75$ & $3.63 \pm 0.61$ & $3.69 \pm 0.64$ & 0.394 \\
& SER & $3.99 \pm 0.51$ & $3.91 \pm 0.58$ & $3.77 \pm 0.50$ & $3.89 \pm 0.54$ & 0.184 \\
& SET & $3.43 \pm 0.62$ & $3.50 \pm 0.56$ & $3.39 \pm 0.45$ & $3.44 \pm 0.55$ & 0.671 \\
\multirow{2}{*}{ AFTT } & FTF & $3.58 \pm 1.05 \triangle$ & $2.94 \pm 0.96 \nabla$ & $3.09 \pm 1.08$ & $3.20 \pm 1.06$ & 0.011 \\
& FTB & $3.98 \pm 0.84$ & $3.96 \pm 0.64$ & $3.91 \pm 0.62$ & $3.95 \pm 0.70$ & 0.924 \\
& FTT & $3.53 \pm 0.45$ & $3.62 \pm 0.54$ & $3.40 \pm 0.42$ & $3.52 \pm 0.48$ & 0.121 \\
\hline
\end{tabular}

Values are mean \pm standard deviation.

SEC: Self-confidence scale, SER: Self-regulatory efficacy scale, SET: Task difficulty preference scale, FTF: Feeling scale, FTB: Behavior scale, FTT: Preferred task difficulty scale, The score of $\triangle$ group is significantly higher than that of $\nabla$ group.

"p $<0.05$ (By One way ANOVA test).

Table 3. Subscales Results of Academic Motivation Tests for each Sasang Constitutional Type

\begin{tabular}{ccccccc}
\hline & & Soyangin & Taeeumin & Soeumin & Total & p-value* \\
\hline \multirow{2}{*}{ ASET } & SEC & $3.80 \pm 0.54$ & $3.64 \pm 0.75$ & $3.63 \pm 0.61$ & $3.69 \pm 0.64$ & 0.394 \\
& SER & $3.99 \pm 0.51$ & $3.91 \pm 0.58$ & $3.77 \pm 0.50$ & $3.89 \pm 0.54$ & 0.184 \\
& SET & $3.43 \pm 0.62$ & $3.50 \pm 0.56$ & $3.39 \pm 0.45$ & $3.44 \pm 0.55$ & 0.671 \\
\multirow{2}{*}{ AFTT } & FTF & $3.58 \pm 1.05 \triangle$ & $2.94 \pm 0.96 \nabla$ & $3.09 \pm 1.08$ & $3.20 \pm 1.06$ & 0.011 \\
& FTB & $3.98 \pm 0.84$ & $3.96 \pm 0.64$ & $3.91 \pm 0.62$ & $3.95 \pm 0.70$ & 0.924 \\
& FTT & $3.53 \pm 0.45$ & $3.62 \pm 0.54$ & $3.40 \pm 0.42$ & $3.52 \pm 0.48$ & 0.121 \\
\hline
\end{tabular}

Values are mean \pm standard deviation.

ASEF: Academic Self-efficacy test, AFTT: Academic failure tolerance test. SEC: Self-confidence scale, SER: Self-regulatory efficacy scale, SET: Task difficulty preference scale, FTF: Feeling scale, FTB: Behavior scale, FTT: Preferred task difficulty scale, The score of $\triangle$ group is significantly higher than that of $\nabla$ group.

"p $<0.05$ (By One way ANOVA test). 


\section{A Preliminary Comparison of the Efficacy of Auricular Acupuncture, Transdermal Nicotine Patch and Combination Therapy for Smoking Cessation}

Hee-Chul Kang

J of Oriental Neuropsychiatry 2013:24(2):179-188, http://dx.doi.org/10.7231/jon. 2013.24.2.179

The word " $\mathrm{N}$ " in the legend of Table 1 should have been written as " $\mathrm{n}$ "

"a" Description has been added.

Table 1. General Characteristics of Study Subjects

\begin{tabular}{lcccc}
\hline & $\begin{array}{c}\text { AA group (N=62) } \\
(\text { Mean } \pm \text { S.D. })\end{array}$ & $\begin{array}{c}\text { NP group (N=69) } \\
(\text { Mean } \pm \text { S.D. })\end{array}$ & $\begin{array}{c}\text { AN group (N=57) } \\
(\text { Mean } \pm \text { S.D. })\end{array}$ & p-value \\
\hline Age (years) & $39.53 \pm 8.44^{\mathrm{a}}$ & $37.39 \pm 7.57^{\mathrm{a}}$ & $38.57 \pm 9.50^{\mathrm{a}}$ & 0.35 \\
Height $(\mathrm{cm})$ & $173.37 \pm 7.91^{\mathrm{a}}$ & $172.80 \pm 5.30^{\mathrm{a}}$ & $171.98 \pm 4.38^{\mathrm{a}}$ & 0.46 \\
Weight $(\mathrm{kg})$ & $73.63 \pm 8.58^{\mathrm{a}}$ & $69.33 \pm 6.58^{\mathrm{a}}$ & $67.21 \pm 6.71^{\mathrm{a}}$ & 0.27 \\
Duration of smoking (years) & $17.17 \pm 8.00^{\mathrm{a}}$ & $16.02 \pm 6.73^{\mathrm{a}}$ & $16.68 \pm 8.98^{\mathrm{a}}$ & 0.70 \\
\hline
\end{tabular}

The same superscripts indicate non-significant difference between groups based on Scheffe test.

AA: Auricular Acupuncture, NP: Nicotine Patch, AN: Combination with Auricular Acupuncture and Nicotine Patch, S.D.: Standard deviation, $\mathrm{p}$-value by one way-ANOVA.

"p $<0.05$.

Table 1. General Characteristics of Study Subjects

\begin{tabular}{lcccc}
\hline & $\begin{array}{c}\text { AA group }(\mathrm{n}=62) \\
(\text { Mean } \pm \text { S.D. })\end{array}$ & $\begin{array}{c}\text { NP group }(\mathrm{n}=69) \\
(\text { Mean } \pm \text { S.D. })\end{array}$ & $\begin{array}{c}\text { AN group }(\mathrm{n}=57) \\
(\text { Mean } \pm \text { S.D. })\end{array}$ & p-value \\
\hline Age (years) & $39.53 \pm 8.44^{\mathrm{a}}$ & $37.39 \pm 7.57^{\mathrm{a}}$ & $38.57 \pm 9.50^{\mathrm{a}}$ & 0.35 \\
Height $(\mathrm{cm})$ & $173.37 \pm 7.91^{\mathrm{a}}$ & $172.80 \pm 5.30^{\mathrm{a}}$ & $171.98 \pm 4.38^{\mathrm{a}}$ & 0.46 \\
Weight $(\mathrm{kg})$ & $73.63 \pm 8.58^{\mathrm{a}}$ & $69.33 \pm 6.58^{\mathrm{a}}$ & $67.21 \pm 6.71^{\mathrm{a}}$ & 0.27 \\
Duration of smoking (years) & $17.17 \pm 8.00^{\mathrm{a}}$ & $16.02 \pm 6.73^{\mathrm{a}}$ & $16.68 \pm 8.98^{\mathrm{a}}$ & 0.70 \\
\hline
\end{tabular}

${ }^{a}$ The same superscripts indicate non-significant difference between groups based on Scheffe test.

AA: Auricular Acupuncture, NP: Nicotine Patch, AN: Combination with Auricular Acupuncture and Nicotine Patch, S.D.: Standard deviation, $\mathrm{p}$-value by one way-ANOVA.

*p $<0.05$. 
The word " $\mathrm{N}$ " in the legend of Table 1 should have been written as " $\mathrm{n}$ "

"a" Description has been added.

Table 2. Nicotine Dependence and Amounts of Daily Smoking in First Examination

\begin{tabular}{lcccc}
\hline & $\begin{array}{c}\text { AA group (N=62) } \\
\text { (Mean } \pm \text { S.D. })\end{array}$ & $\begin{array}{c}\text { NP group (N=69) } \\
(\text { Mean } \pm \text { S.D. })\end{array}$ & $\begin{array}{c}\text { AN group (N=57) } \\
\text { (Mean } \pm \text { S.D. })\end{array}$ & p-value \\
\hline Nicotine dependence & $5.67 \pm 2.09^{\mathrm{a}}$ & $5.26 \pm 1.72^{\mathrm{a}}$ & $5.39 \pm 1.76^{\mathrm{a}}$ & 0.43 \\
Amounts of daily smoking (pieces) & $12.95 \pm 6.61^{\mathrm{a}}$ & $12.30 \pm 6.30^{\mathrm{a}}$ & $12.67 \pm 6.20^{\mathrm{a}}$ & 0.84 \\
\hline
\end{tabular}

The same superscripts indicate non-significant difference between groups based on Scheffe test.

AA: Auricular Acupuncture, NP: Nicotine Patch, AN: Combination with Auricular Acupuncture and Nicotine Patch, S.D.: Standard deviation, $\mathrm{p}$-value by one way-ANOVA. *p $<0.05$.

Table 2. Nicotine Dependence and Amounts of Daily Smoking in First Examination

\begin{tabular}{lcccc}
\hline & $\begin{array}{c}\text { AA group (n=62) } \\
\text { (Mean } \pm \text { S.D. })\end{array}$ & $\begin{array}{c}\text { NP group (n=69) } \\
(\text { Mean } \pm \text { S.D. })\end{array}$ & $\begin{array}{c}\text { AN group }(\mathrm{n}=57) \\
(\text { Mean } \pm \text { S.D. })\end{array}$ & p-value \\
\hline Nicotine dependence & $5.67 \pm 2.09^{\mathrm{a}}$ & $5.26 \pm 1.72^{\mathrm{a}}$ & $5.39 \pm 1.76^{\mathrm{a}}$ & 0.43 \\
Amounts of daily smoking (pieces) & $12.95 \pm 6.61^{\mathrm{a}}$ & $12.30 \pm 6.30^{\mathrm{a}}$ & $12.67 \pm 6.20^{\mathrm{a}}$ & 0.84 \\
\hline
\end{tabular}

${ }^{a}$ The same superscripts indicate non-significant difference between groups based on Scheffe test.

AA: Auricular Acupuncture, NP: Nicotine Patch, AN: Combination with Auricular Acupuncture and Nicotine Patch, S.D.: Standard deviation, p-value by one way-ANOVA. "p $<0.05$.

The word " $\mathrm{N}$ " in the legend of Table 1 should have been written as " $\mathrm{n}$ "

Table 3. Comparisons of Nicotine Dependence before and after Treatment

\begin{tabular}{cccc}
\hline & $\begin{array}{c}\text { Before } \\
\text { treatment }\end{array}$ & $\begin{array}{c}\text { After } \\
\text { treatment }\end{array}$ & p-value \\
\hline $\begin{array}{c}\text { AA group (N=62) } \\
(\text { Mean } \pm \text { S.D. })\end{array}$ & $5.67 \pm 2.09$ & $3.58 \pm 2.06$ & $<0.01^{\dagger}$ \\
$\begin{array}{c}\text { NP group (N=69) } \\
\text { (Mean } \pm \text { S.D. })\end{array}$ & $5.26 \pm 1.72$ & $3.17 \pm 1.75$ & $<0.01^{\dagger}$ \\
$\begin{array}{c}\text { AN group (N=57) } \\
(\text { Mean } \pm \text { S.D. })\end{array}$ & $5.39 \pm 1.76$ & $2.73 \pm 1.79$ & $<0.01^{\dagger}$ \\
\hline
\end{tabular}

AA: Auricular Acupuncture, NP: Nicotine Patch, AN: Combination with Auricular Acupuncture and Nicotine Patch, S.D.: Standard deviation, p-value by paired-T test.

"p $<0.05,{ }^{\dagger} \mathrm{p}<0.01$.
Table 3. Comparisons of Nicotine Dependence before and after Treatment

\begin{tabular}{|c|c|c|c|}
\hline & $\begin{array}{c}\text { Before } \\
\text { treatment }\end{array}$ & $\begin{array}{l}\text { After } \\
\text { treatment }\end{array}$ & $\mathrm{p}$-value \\
\hline $\begin{array}{l}\text { AA group }(\mathrm{n}=62) \\
(\text { Mean } \pm \text { S.D. })\end{array}$ & $5.67 \pm 2.09$ & $3.58 \pm 2.06$ & $<0.01^{\dagger}$ \\
\hline $\begin{array}{l}\text { NP group }(n=69) \\
(\text { Mean } \pm \text { S.D. })\end{array}$ & $5.26 \pm 1.72$ & $3.17 \pm 1.75$ & $<0.01^{\dagger}$ \\
\hline $\begin{array}{l}\text { AN group }(n=57) \\
(\text { Mean } \pm \text { S.D. })\end{array}$ & $5.39 \pm 1.76$ & $2.73 \pm 1.79$ & $<0.01^{\dagger}$ \\
\hline \multicolumn{4}{|c|}{$\begin{array}{l}\text { AA: Auricular Acupuncture, NP: Nicotine Patch, AN: } \\
\text { Combination with Auricular Acupuncture and Nicotine } \\
\text { Patch, S.D.: Standard deviation, p-value by paired-T } \\
\text { test. } \\
{ }^{*} \mathrm{p}<0.05,{ }^{\dagger} \mathrm{p}<0.01 \text {. }\end{array}$} \\
\hline
\end{tabular}


The word " $\mathrm{N}$ " in the legend of Table 1 should have been written as " $\mathrm{n}$ "

Table 4. Comparisons of Amounts of Daily Smoking before and after Treatment

\begin{tabular}{cccc}
\hline & $\begin{array}{c}\text { Pre- } \\
\text { treatment }\end{array}$ & $\begin{array}{c}\text { Post- } \\
\text { treatment }\end{array}$ & p-value \\
\hline $\begin{array}{c}\text { AA group (N=62) } \\
(\text { Mean } \pm \text { S.D. })\end{array}$ & $12.95 \pm 6.61$ & $7.65 \pm 5.20$ & $<0.01^{\dagger}$ \\
$\begin{array}{c}\text { NP group (N=69) } \\
(\text { Mean } \pm \text { S.D. })\end{array}$ & $12.30 \pm 6.30$ & $6.49 \pm 4.54$ & $<0.01^{\dagger}$ \\
$\begin{array}{c}\text { AN group (N=57) } \\
(\text { Mean } \pm \text { S.D.) }\end{array}$ & $12.67 \pm 6.20$ & $6.26 \pm 4.47$ & $<0.01^{\dagger}$ \\
\hline
\end{tabular}

AA: Auricular Acupuncture, NP: Nicotine Patch, AN: Combination with Auricular Acupuncture and Nicotine Patch, S.D.: Standard deviation, p-value by paired-T test.

"p $<0.05^{\dagger} \mathrm{p}<0.01$.
Table 4. Comparisons of Amounts of Daily Smoking before and after Treatment

\begin{tabular}{|c|c|c|c|}
\hline & $\begin{array}{l}\text { Pre- } \\
\text { treatment }\end{array}$ & $\begin{array}{c}\text { Post- } \\
\text { treatment }\end{array}$ & p-value \\
\hline $\begin{array}{c}\text { AA group }(\mathrm{n}=62) \\
(\text { Mean } \pm \text { S.D. })\end{array}$ & $12.95 \pm 6.61$ & $7.65 \pm 5.20$ & $<0.01^{\dagger}$ \\
\hline $\begin{array}{l}\text { NP group }(\mathrm{n}=69) \\
(\text { Mean } \pm \text { S.D. })\end{array}$ & $12.30 \pm 6.30$ & $6.49 \pm 4.54$ & $<0.01^{\dagger}$ \\
\hline $\begin{array}{c}\text { AN group }(n=57) \\
(\text { Mean } \pm \text { S.D. })\end{array}$ & $12.67 \pm 6.20$ & $6.26 \pm 4.47$ & $<0.01^{\dagger}$ \\
\hline
\end{tabular}

AA: Auricular Acupuncture, NP: Nicotine Patch, AN: Combination with Auricular Acupuncture and Nicotine Patch, S.D.: Standard deviation, p-value by paired-T test.

${ }^{*} \mathrm{p}<0.05,{ }^{\dagger} \mathrm{p}<0.01$

The word " $\mathrm{N}$ " in the legend of Table 1 should have been written as " $\mathrm{n}$ " "a", "b" Description has been added.

Table 5. Decrements of Nicotine Dependence and Amounts of Daily Smoking

\begin{tabular}{|c|c|c|c|c|}
\hline Decrements & $\begin{array}{l}\text { AA group }(\mathrm{N}=62) \\
\quad(\text { Mean } \pm \text { S.D. })\end{array}$ & $\begin{array}{l}\text { NP group }(\mathrm{N}=69) \\
\quad(\text { Mean } \pm \text { S.D. })\end{array}$ & $\begin{array}{l}\text { AN group }(\mathrm{N}=57) \\
\quad(\text { Mean } \pm \text { S.D. })\end{array}$ & p-value \\
\hline Nicotine dependence & $2.10 \pm 1.17^{\mathrm{a}}$ & $2.09 \pm 1.16^{\mathrm{a}}$ & $2.65 \pm 1.29^{\mathrm{b}}$ & $0.02^{*}$ \\
\hline Amounts of daily smoking (pieces) & $5.39 \pm 4.01^{\mathrm{a}}$ & $5.81 \pm 4.16^{\mathrm{a}}$ & $6.40 \pm 4.90^{\mathrm{a}}$ & 0.44 \\
\hline
\end{tabular}

The same superscripts indicate non-significant difference between groups based on Scheffe test.

AA: Auricular Acupuncture, NP: Nicotine Patch, AN: Combination with Auricular Acupuncture and Nicotine Patch, S.D.: Standard deviation, p-value by one way-ANOVA. *p $<0.05$.

Table 5. Decrements of Nicotine Dependence and Amounts of Daily Smoking

\begin{tabular}{|c|c|c|c|c|}
\hline Decrements & $\begin{array}{l}\text { AA group }(\mathrm{n}=62) \\
\quad(\text { Mean } \pm \text { S.D. })\end{array}$ & $\begin{array}{l}\text { NP group }(n=69) \\
(\text { Mean } \pm \text { S.D. })\end{array}$ & $\begin{array}{l}\text { AN group }(\mathrm{n}=57) \\
\quad(\text { Mean } \pm \text { S.D. })\end{array}$ & $\mathrm{p}$-value \\
\hline Nicotine dependence & $2.10 \pm 1.17^{\mathrm{a}}$ & $2.09 \pm 1.16^{\mathrm{a}}$ & $2.65 \pm 1.29^{\mathrm{b}}$ & $0.02 *$ \\
\hline Amounts of daily smoking (pieces) & $5.39 \pm 4.01^{\mathrm{a}}$ & $5.81 \pm 4.16^{\mathrm{a}}$ & $6.40 \pm 4.90^{\mathrm{a}}$ & 0.44 \\
\hline
\end{tabular}

${ }^{a}$ The same superscripts indicate non-significant difference between groups based on Scheffe test. ${ }^{\mathrm{b}}$ The different superscripts indicate significant difference between groups based on Scheffe test.

AA: Auricular Acupuncture, NP: Nicotine Patch, AN: Combination with Auricular Acupuncture and Nicotine Patch, S.D.: Standard deviation, p-value by one way-ANOVA. $* \mathrm{p}<0.05$. 


\section{The Effects of OnDam-tang-Kami-bang (ODK) in Antioxidant and Serotonin Metabolism Testing on P815 Cell}

Seon-Hui Seol, Sang Ryong Lee, In Chul Jung

J of Oriental Neuropsychiatry 2013:24(2):189-200, http://dx. doi.org/10.7231/jon.2013.24.2.189

Added the abbreviation explanation of the "DPPH"
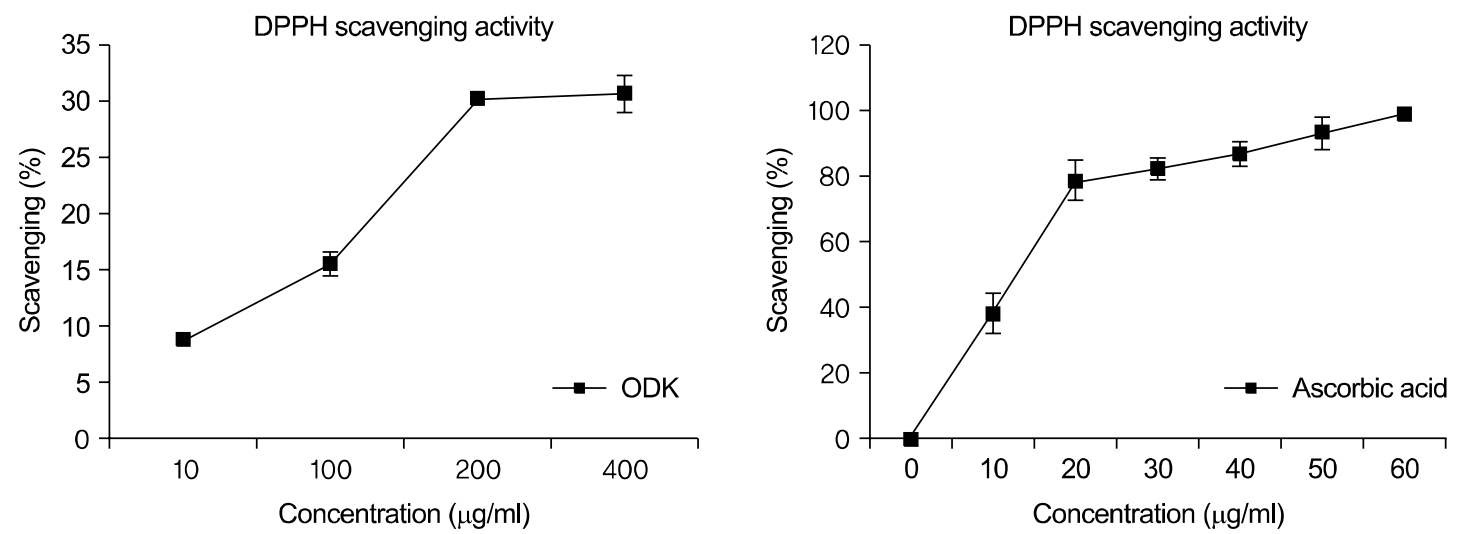

Fig. 2. Effect of OnDam-tang-Kami-bang (ODK) and ascorbic acid on DPPH radical-scavenging activity. DPPH scavenging activity was measured as described in Material and Methods. Date are expressed as \% of scavening and each column represents the mean \pm SD $(n>3)$.
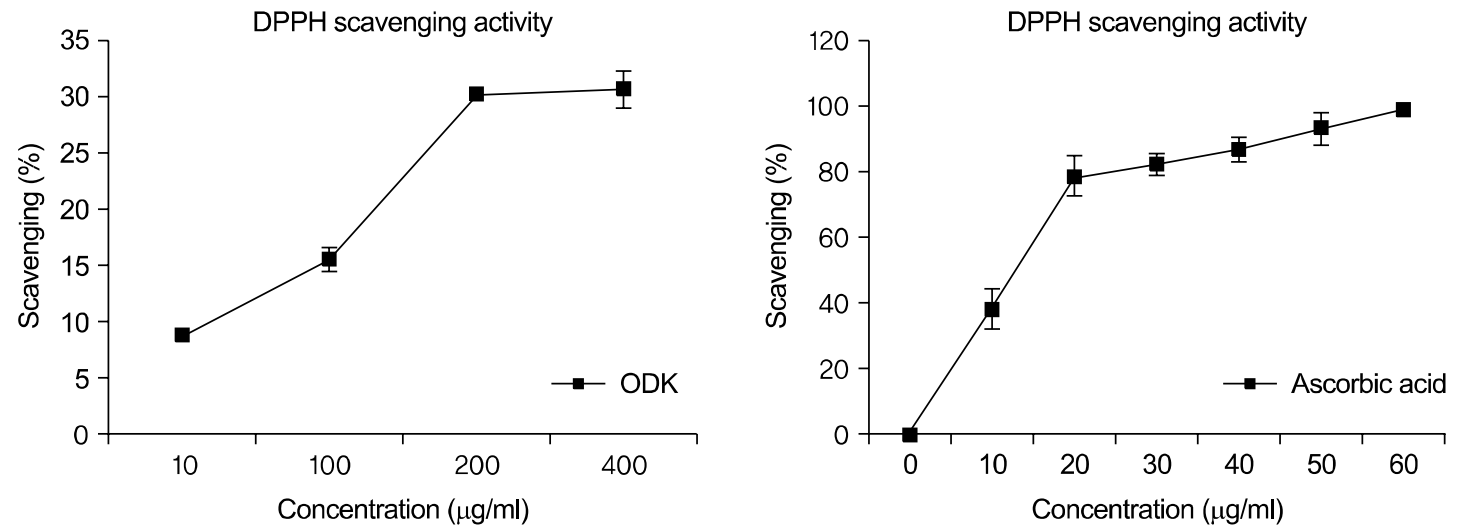

Fig. 2. Effect of OnDam-tang-Kami-bang (ODK) and ascorbic acid on DPPH radical-scavenging activity. DPPH scavenging activity was measured as described in Material and Methods. Date are expressed as \% of scavening and each column represents the mean $\pm S D(n>3)$.

DPPH: 2,2-Diphenyl-1-picrylhydrazyl. 
Added the abbreviation explanation of the "DPPH"

Table 3. Effect of OnDam-tang-Kami-bang (ODK) on DPPH Radical-scavenging Activity

\begin{tabular}{ccc}
\hline Sample & Concentraion & Scavenging effect (\%) \\
\hline \multirow{4}{*}{ Ascorbic acid } & $60 \mu \mathrm{g} / \mathrm{ml}$ & $99.3 \pm 1.15$ \\
& $50 \mu \mathrm{g} / \mathrm{ml}$ & $93.7 \pm 4.93$ \\
& $40 \mu \mathrm{g} / \mathrm{ml}$ & $87.0 \pm 4.00$ \\
& $30 \mu \mathrm{g} / \mathrm{ml}$ & $82.7 \pm 3.51$ \\
& $20 \mu \mathrm{g} / \mathrm{ml}$ & $79.0 \pm 6.08$ \\
& $10 \mu \mathrm{g} / \mathrm{ml}$ & $38.0 \pm 6.08$ \\
\hline \multirow{4}{*}{ ODK } & $400 \mu \mathrm{g} / \mathrm{ml}$ & $30.8 \pm 1.65$ \\
& $200 \mu \mathrm{g} / \mathrm{ml}$ & $30.4 \pm 0.88$ \\
& $100 \mu \mathrm{g} / \mathrm{ml}$ & $15.6 \pm 1.04$ \\
& $10 \mu \mathrm{g} / \mathrm{ml}$ & $8.7 \pm 0.52$ \\
\hline
\end{tabular}

Added the abbreviation explanation of the "SOD"

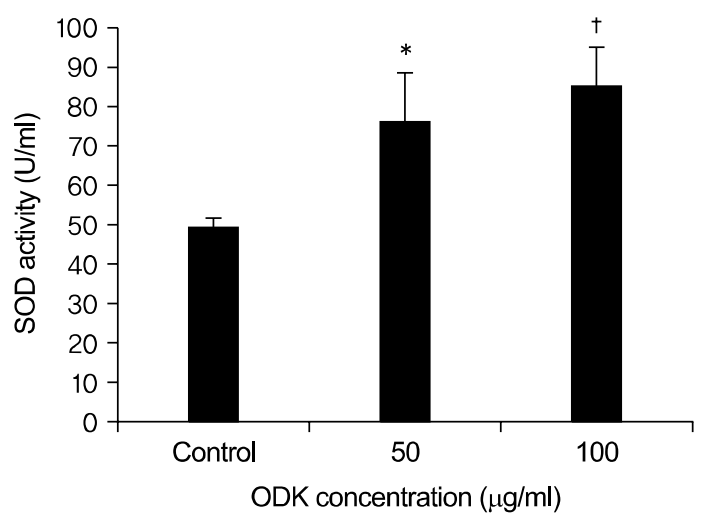

Fig. 3. Effect of OnDam-tang-Kami-bang (ODK) on the SOD activity. The effect on SOD was tested with ODK, date are expressed as \% of control and each column represents the mean $\pm S D$ of two determination. Statistically significant value compared with control by $T$ test $\left({ }^{*} p<0.05,{ }^{\dagger} p<0.01\right)$.
Table 3. Effect of OnDam-tang-Kami-bang (ODK) on DPPH Radical-scavenging Activity

\begin{tabular}{ccc}
\hline Sample & Concentraion & Scavenging effect (\%) \\
\hline \multirow{4}{*}{ Ascorbic acid } & $60 \mu \mathrm{g} / \mathrm{ml}$ & $99.3 \pm 1.15$ \\
& $50 \mu \mathrm{g} / \mathrm{ml}$ & $93.7 \pm 4.93$ \\
& $30 \mu \mathrm{g} / \mathrm{ml}$ & $87.0 \pm 4.00$ \\
& $20 \mu \mathrm{g} / \mathrm{ml}$ & $82.7 \pm 3.51$ \\
& $10 \mu \mathrm{g} / \mathrm{ml}$ & $79.0 \pm 6.08$ \\
\hline \multirow{4}{*}{ ODK } & $400 \mu \mathrm{g} / \mathrm{ml}$ & $38.0 \pm 6.08$ \\
\hline & $200 \mu \mathrm{g} / \mathrm{ml}$ & $30.8 \pm 1.65$ \\
& $100 \mu \mathrm{g} / \mathrm{ml}$ & $15.6 \pm 1.04$ \\
& $10 \mu \mathrm{g} / \mathrm{ml}$ & $8.7 \pm 0.52$ \\
\hline
\end{tabular}

DPPH: 2,2-Diphenyl-1-picrylhydrazyl.

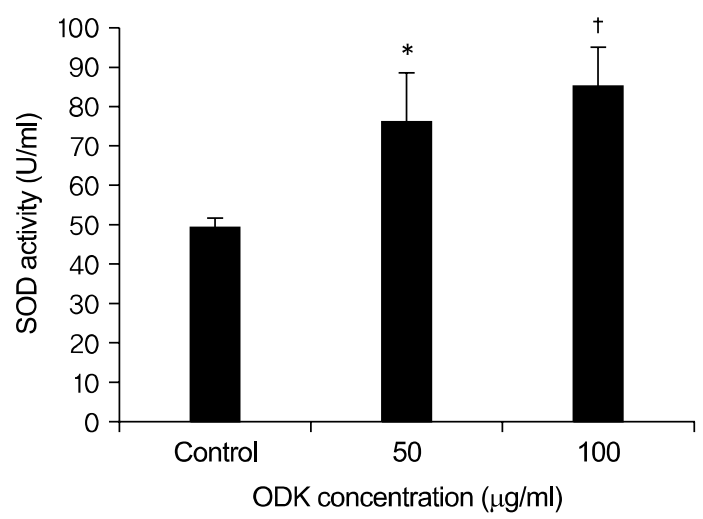

Fig. 3. Effect of OnDam-tang-Kami-bang (ODK) on the SOD activity. The effect on SOD was tested with ODK, date are expressed as \% of control and each column represents the mean $\pm S D$ of two determination. Statistically significant value compared with control by $T$ test $\left({ }^{*} p<0.05,{ }^{\dagger} p<0.01\right)$. SOD: Superoxide dismutase. 
Added the abbreviation explanation of the "HTT"

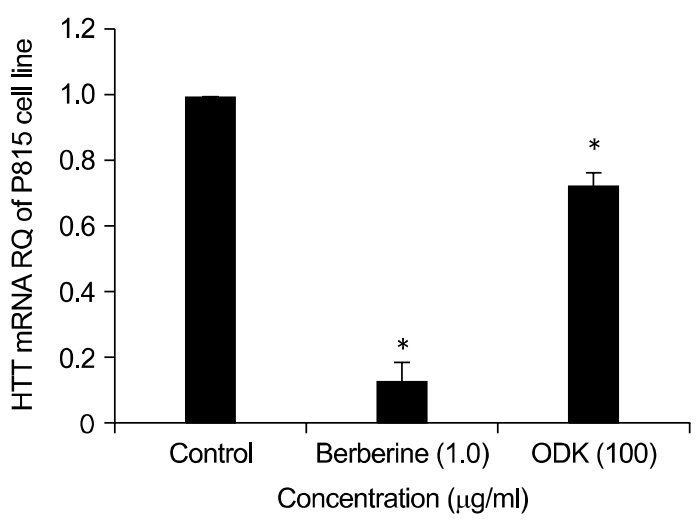

Fig. 5. Effect of OnDam-tang-Kami-bang (ODK) on 5-HTT mRNA in P815 cells. The expression levels of $5-\mathrm{HTT}$ mRNA and beta-actin were analyzed by real-time RT-PCR. The 5-HTT mRNA expression was normalized to beta-actin mRNA expression in the corresponding sample. Values are means \pm SEM $\left({ }^{*} \mathrm{p}<0.001\right)$.

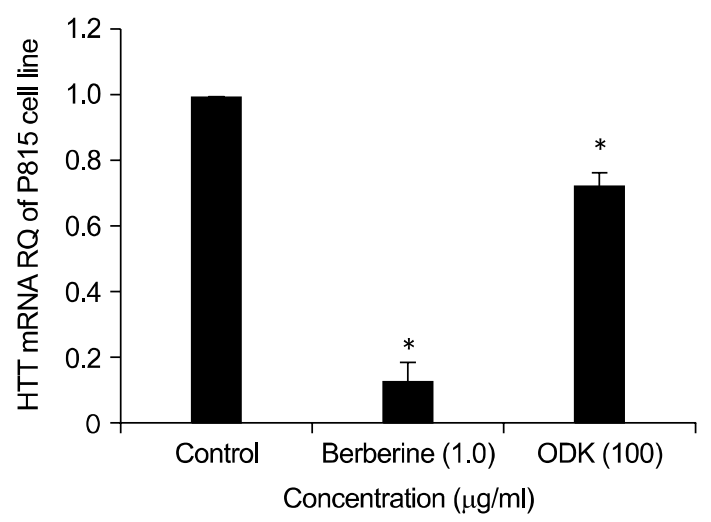

Fig. 5. Effect of OnDam-tang-Kami-bang (ODK) on 5-HTT mRNA in P815 cells. The expression levels of 5-HTT mRNA and beta-actin were analyzed by real-time RT-PCR. The 5-HTT mRNA expression was normalized to beta-actin mRNA expression in the corresponding sample. Values are means \pm SEM $\left({ }^{*} \mathrm{p}<0.001\right)$.

HTT: hydroxytryptamine transporte.

Added the abbreviation explanation of the "5-HTT"

Table 5. Effect of OnDam-tang-Kami-bang (ODK) on 5-HTT mRNA in P815 Cells

\begin{tabular}{ll}
\hline \multicolumn{1}{c}{ Compounds } & $\begin{array}{c}\text { mRNA expression } \\
(\% \text { of control })\end{array}$ \\
\hline Control & $0.996 \pm 0.005(100)$ \\
Berberine $(3 \mu \mathrm{M})$ & $0.129 \pm 0.056(13.0)^{*}$ \\
ODK $(100 \mu \mathrm{g} / \mathrm{ml})$ & $0.722 \pm 0.036(72.4)^{*}$ \\
\hline
\end{tabular}

Table 5. Effect of OnDam-tang-Kami-bang (ODK) on $5-\mathrm{HTT}$ mRNA in P815 Cells

\begin{tabular}{ll}
\hline \multicolumn{1}{c}{ Compounds } & $\begin{array}{c}\text { mRNA expression } \\
(\% \text { of control })\end{array}$ \\
\hline Control & $0.996 \pm 0.005(100)$ \\
Berberine $(3 \mu \mathrm{M})$ & $0.129 \pm 0.056(13.0)^{* *}$ \\
ODK $(100 \mu \mathrm{g} / \mathrm{ml})$ & $0.722 \pm 0.036(72.4)^{*}$ \\
\hline
\end{tabular}

$* \mathrm{p}<0.001$.

5-HTT: 5-hydroxytryptamine transporte. 
Added the abbreviation explanation of the "TPH-1"

Table 6. Effect of OnDam-tang-Kami-bang (ODK) on TPH-1 mRNA in P815 Cells

\begin{tabular}{ll}
\hline \multicolumn{1}{c}{ Compounds } & $\begin{array}{c}\text { mRNA expression } \\
(\% \text { of control })\end{array}$ \\
\hline Control & $0.996 \pm 0.005(100)$ \\
Berberine $(3 \mu \mathrm{M})$ & $0.159 \pm 0.050(16.0)^{\dagger}$ \\
ODK $(100 \mu \mathrm{g} / \mathrm{ml})$ & $0.766 \pm 0.067(76.9)^{*}$ \\
\hline${ }^{\mathrm{p}}<<0.01,{ }^{\dagger} \mathrm{p}<0.001$. &
\end{tabular}

Table 6. Effect of OnDam-tang-Kami-bang (ODK) on TPH-1 mRNA in P815 Cells

\begin{tabular}{ll}
\hline \multicolumn{1}{c}{ Compounds } & $\begin{array}{c}\text { mRNA expression } \\
(\% \text { of control })\end{array}$ \\
\hline Control & $0.996 \pm 0.005(100)$ \\
Berberine $(3 \mu \mathrm{M})$ & $0.159 \pm 0.050(16.0)^{\dagger}$ \\
ODK $(100 \mu \mathrm{g} / \mathrm{ml})$ & $0.766 \pm 0.067(76.9)^{*}$ \\
\hline "p $<0.01,{ }^{\dagger} \mathrm{p}<0.001$. \\
TPH-1: Tryptophan hydroxylase-1.
\end{tabular}

Added the abbreviation explanation of the "TPH-1", "RT-PCR", "SEM"

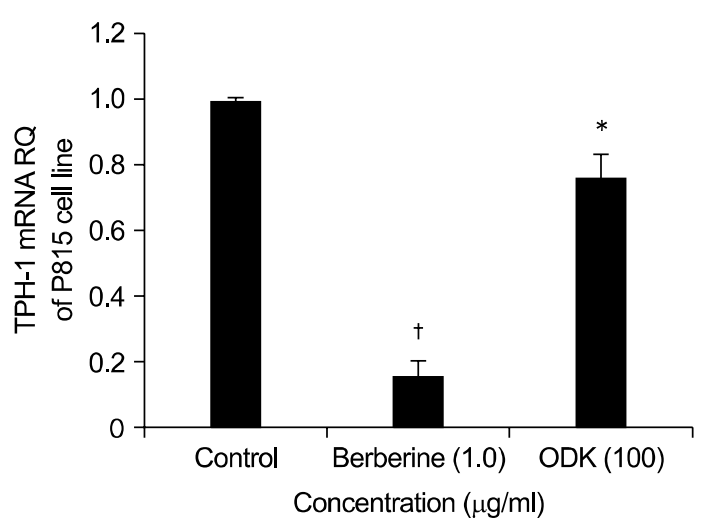

Fig. 6. Effect of OnDam-tang-Kami-bang (ODK) on TPH-1 mRNA in P815 cells. The expression levels of TPH-1 mRNA and beta-actin were analyzed by real-time RT-PCR. The TPH-1 mRNA expression was normalized to beta-actin mRNA expression in the corresponding sample. Values are means \pm SEM $\left({ }^{*} p<0.01,{ }^{\dagger} p<0.001\right)$.

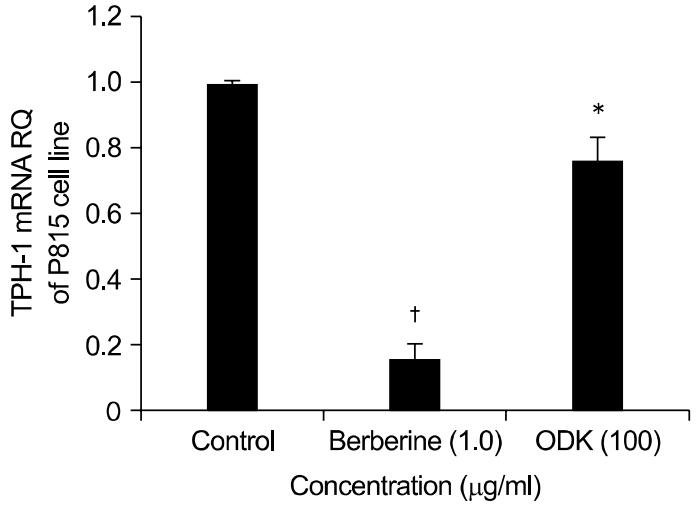

Fig. 6. Effect of OnDam-tang-Kami-bang (ODK) on TPH-1 mRNA in P815 cells. The expression levels of TPH-1 mRNA and beta-actin were analyzed by real-time RT-PCR. The TPH-1 mRNA expression was normalized to beta-actin mRNA expression in the corresponding sample. Values are means \pm SEM $\left({ }^{*} p<0.01,{ }^{\dagger} p<0.001\right)$.

TPH-1: Tryptophan hydroxylase-1, RT-PCR: real time polymerase chain reaction, SEM: Standard Error of the Mean. 